\section{A New Approach to Teaching Trauma Assessment and Management to Paramedic Students}

\author{
*David R. Johnson, MD, FACEP, Darryl Macias, \\ MD, Ann Dunlap, PhD, NREMT-P, Mark \\ Hauswald, $M D$ \\ New Mexico Emergency Medical Services Academy, \\ Department of Emergency-Medicine, University of New \\ Mexico, Albuquerque, New Mexico
}

Purpose: To determine if teaching EMT-P students to prioritize on-scene procedures (OSPs) will affect scene time and the appropriateness of OSPs.

Methods: Paramedic student performances during scenario-based testing were recorded on videotape. In 1992, a new trauma curriculum was introduced that stressed the prioritization of OSPs. Procedures included: spinal immobilization, MAST, intubation, cricothyrotomy, IVs, and chest decompression. Random sampling of $20 \mathrm{stu}-$ dent performances from the 1993 class were compared to a sample of 20 from the 1991 class. Scene times and number of OSPs were measured from the video tape. Two independent, EMS physician reviewers evaluated the appropriateness of scene times on a linear analog scale. They also recorded the number of inappropriate OSPs. The data are presented as mean \pm sd and analyzed using the $t$-test and ANOVA.

Results: Scene time from 1991 to 1993 decreased: $629.1 \pm 83.8 \mathrm{~min}$ vs. $371.6 \pm 273.8 \mathrm{~min}(p<0.001)$ as $\mathrm{did}$ the number of OSPs: $3.1 \pm 0.6$ vs. $1.7 \pm 1.6(p<0.001)$. Improvement in the appropriateness of scene time from 1991 to 1993 was noted by the physician reviewers.

Reviewer 1: $21.2 \pm 18.7 \mathrm{~mm}$ vs. $66.2 \pm 29.7 \mathrm{~mm}(p<0.001)$. Reviewer 2: $21.7 \pm 17.1 \mathrm{~mm}$ vs. $59.5 \pm 29.5 \mathrm{~mm}(p<0.001)$.

A significant difference in the number of inappropriate OSPs was noted between the two groups.

Reviewer $1: 1.4 \pm 1.2$ vs. $0.3 \pm 0.7(p<0.001)$. Reviewer $2: 1.6 \pm 0.9$ vs. $0.5 \pm 0.7(p<0.001)$.

Conclusions: Using a new curriculum, which stresses current prehospital research, scene time and on-scene procedures can be reduced.

\section{Lack of Interrater Angreement when Scoring NAEMSP Abstracts}

\author{
*Thomas E. Auble, PhD \\ Division of Emergency Medicine;University of Pittsburgh \\ School of Medicine, Pittsburgh, Pennsylvania
}

Purpose: Each year, researchers submit abstracts for presentation at one or more emergency medicine annual meetings. The decision to accept or reject an abstract for presentation and the presentation format for submissions to the NAEMSP Annual Meeting is determined on the basis of scores assigned by several expert reviewers. Some degree of agreement among reviewer scores is desirable to help ensure that the selection process is reliable and not a function of chance. Whether the scoring of abstracts for these meetings is reliable or not is unknown.

Hypothesis: There is limited agreement among reviewer scores of abstracts submitted for the NAEMSP Annual Meeting.

Methods: A total of 84 abstracts were submitted. The abstracts were scored by the review chairman and three other selected expert reviewers. Possible scores ranged from 1 ( unacceptable) to 5 (excellent). Scores from all reviewers were averaged to obtain a final score. Reviewers abstained from scoring abstracts when a potential conflict of interest occurred. Interrater agreement was determined using the Kappa statistic for the 68 abstracts scored by all reviewers. Median scores for each reviewer were compared by Kruskal-Wallis ANOVA. The alpha error was set at 0.05 .

Results: The distribution of scores assigned by each reviewer was as follows:

\begin{tabular}{|c|c|c|c|c|c|c|}
\hline \multirow[t]{2}{*}{ Reviewer } & \multicolumn{5}{|c|}{ Distribution of Scores } & \multirow{2}{*}{$\begin{array}{l}\text { Median } \\
\text { Scores }\end{array}$} \\
\hline & 1 & 2 & 3 & 4 & 5 & \\
\hline 1 & 25 & 32 & 10 & 1 & 0 & 2 \\
\hline 2 & 15 & 25 & 23 & 5 & 0 & 2 \\
\hline 3 & 3 & 16 & 26 & 21 & 2 & 3 \\
\hline 4 & 7 & 14 & 12 & 16 & 19 & 4 \\
\hline
\end{tabular}

The overall interrater agreement was 0.04 with a standard error of 0.03 . The level of agreement was not significant $(p=0.06)$. The highest level of agreement occurred between reviewers 1 and 2, with a Kappa statistic of $0.22(p<0.01)$. There was a significant difference in median scores among reviewers $(p<0.01)$.

Conclusions: The agreement among reviewers of the 1993 NAEMSP Annual Meeting abstracts was well below the 0.04 threshold generally regarded to represent more than poor agreement beyond chance. Methods designed to improve reviewer agreement including alternate scoring systems, reviewer training, and selection of reviewers should be considered in the future. 